\title{
Evaluating Bag-of-Visual-Words Representations in Scene Classification
}

\author{
Jun Yang \\ School of Computer Science \\ Carnegie Mellon University \\ Pittsburgh, PA 15213 \\ juny@cs.cmu.edu \\ Alexander G. Hauptmann \\ School of Computer Science \\ Carnegie Mellon University \\ Pittsburgh, PA 15213 \\ alex@cs.cmu.edu
}

\author{
Yu-Gang Jiang \\ Dept of Computer Science \\ City University of Hong Kong \\ Kowloon, Hong Kong \\ yjiang@cs.cityu.edu.hk \\ Chong-Wah Ngo \\ Dept of Computer Science \\ City University of Hong Kong \\ Kowloon, Hong Kong \\ cwngo@cs.cityu.edu.hk
}

\begin{abstract}
Based on keypoints extracted as salient image patches, an image can be described as a "bag of visual words" and this representation has been used in scene classification. The choice of dimension, selection, and weighting of visual words in this representation is crucial to the classification performance but has not been thoroughly studied in previous work. Given the analogy between this representation and the bag-of-words representation of text documents, we apply techniques used in text categorization, including term weighting, stop word removal, feature selection, to generate image representations that differ in the dimension, selection, and weighting of visual words. The impact of these representation choices to scene classification is studied through extensive experiments on the TRECVID and PASCAL collection. This study provides an empirical basis for designing visual-word representations that are likely to produce superior classification performance.
\end{abstract}

\section{Categories and Subject Descriptors}

H.3.3 [Information Storage and Retrieval]: Information Search and Retrieval

\section{General Terms}

Experimentation, Performance

\section{Keywords}

scene classification, keypoint, local interest point, bag-ofvisual-words

Permission to make digital or hard copies of all or part of this work for personal or classroom use is granted without fee provided that copies are not made or distributed for profit or commercial advantage and that copies bear this notice and the full citation on the first page. To copy otherwise, to republish, to post on servers or to redistribute to lists, requires prior specific permission and/or a fee.

MIR'07, September 28-29, 2007, Augsburg, Bavaria, Germany.

Copyright 2007 ACM 978-1-59593-778-0/07/0009 ...\$5.00.

\section{INTRODUCTION}

Classifying images or video scenes into semantic categories is a problem of great interest in both research and practice. For example, an online collection of photos needs to be grouped into categories like "landscape", "portrait", and "animal" to support efficient browsing. To search over a large archive of news video, we want to classify video frames by the presence of certain scenes (e.g., meeting) and objects (e.g., buildings) and by semantic topics (e.g., politics). Scene classification is typically based on real-valued feature vectors describing the color, texture, and other visual properties of images. This representation is significantly different from the sparse and discrete term-vector document representation in text categorization, and therefore, there has been little connection between the two streams of research.

Recently, there is a trend of using image keypoints or local interest points in image retrieval and classification [8, $9,5,18,23,22]$. Keypoints are salient image patches that contain rich local information of an image, and they can be automatically detected using various detectors $[12,22]$ and represented by many descriptors [13]. Keypoints are then grouped into a large number of clusters so that those with similar descriptors are assigned into the same cluster. By treating each cluster as a "visual word" that represents the specific local pattern shared by the keypoints in that cluster, we have a visual-word vocabulary describing all kinds of local image patterns. With its keypoints mapped into visual words, an image can be represented as a "bag of visual words", or specifically, as a vector containing the (weighted) count of each visual word in that image, which can be used as a feature vector in classification task.

This visual-word image representation is analogous to the bag-of-words representation of text documents in terms of form and semantics. This makes techniques for text categorization readily applicable to the problem of scene classification. In this paper, we use text categorization techniques, including term weighting and normalization, stop word removal, and feature selection, to generate image representations with different dimension, selection, and weighting of visual words and study their effectiveness in scene classification tasks. The goal is to provide a missing link in the previous work, where most of the effort has been on various 
keypoint detectors, keypoint descriptors, and clustering and classification algorithms $[8,9,5,18,23,22]$. In comparison, this paper focuses on the representation choices of the visual-word features, which are critical to the classification performance but yet to be thoroughly studied. By evaluating various representation choices, we intend to answer the question of what visual-word representation choices (w.r.t dimension, weighting, selection, etc) are likely to produce the best classification performance in terms of accuracy and efficiency.

We evaluate the image classification performance based on various visual-word representations generated by text categorization techniques on two benchmark corpora, TRECVID and PASCAL. The experiments lead to the following important observations: (1) the size of an effective visual-word vocabulary varies from thousands to tens of thousands; (2) binary visual-word features are as effective as $t f$ or $t f-i d f$ weighted features; (3) using selection criteria such as chisquare and mutual information, half of the visual words in the vocabulary can be eliminated with minimum loss of classification performance; (4) frequent visual words are usually very informative and must not be removed; (5) the spatial information of keypoints is helpful under small vocabularies. These observations are critical to designing the most effective visual-word representation for image classification and other related tasks. We also study the performance obtained by combining visual-word features with conventional color/texture features, from which we find the two types of features are complementary.

In Section 2, we briefly review the existing works on image classification and text categorization. We describe the generation of bag-of-visual-words image representation in Section 3, and discuss the text categorization techniques for generating various representations in Section 4. We introduce the testing corpora and explore the distribution of visual words in Section 5. The experiment results and conclusions are presented in Section 6 and Section 7, respectively.

\section{RELATED WORK}

Representing images by effective features is crucial to the performance of image retrieval and classification. The most popular image representation has been the low-level visual features, which describes an image by the global distribution of color, texture, or other properties. Features like color histograms and Gabor filters belong to this category. To include spatial information, an image is partitioned into either rectangular regions or segments of objects and backgrounds, and features computed from these regions/segments are concatenated into a single image feature vector. These conventional image representations are in the form of real-valued feature vectors, which is different from the sparse term vectors representing text documents.

Recently, the computer vision community has found keypoints to be an effective image representation for tasks varying from object recognition to image classification. Keypoints are salient image patches that contain rich local information of an image. They can be automatically detected using various keypoint detectors, which are surveyed in [12] and [22]. Keypoints are depicted by descriptors like SIFT (scale-invariant feature transform) [11] and its variant PCASIFT [7]. The keypoint descriptors are surveyed in [13]. Keypoint features can be used in their raw format for direct image matching [23], or vector-quantized into a repre-

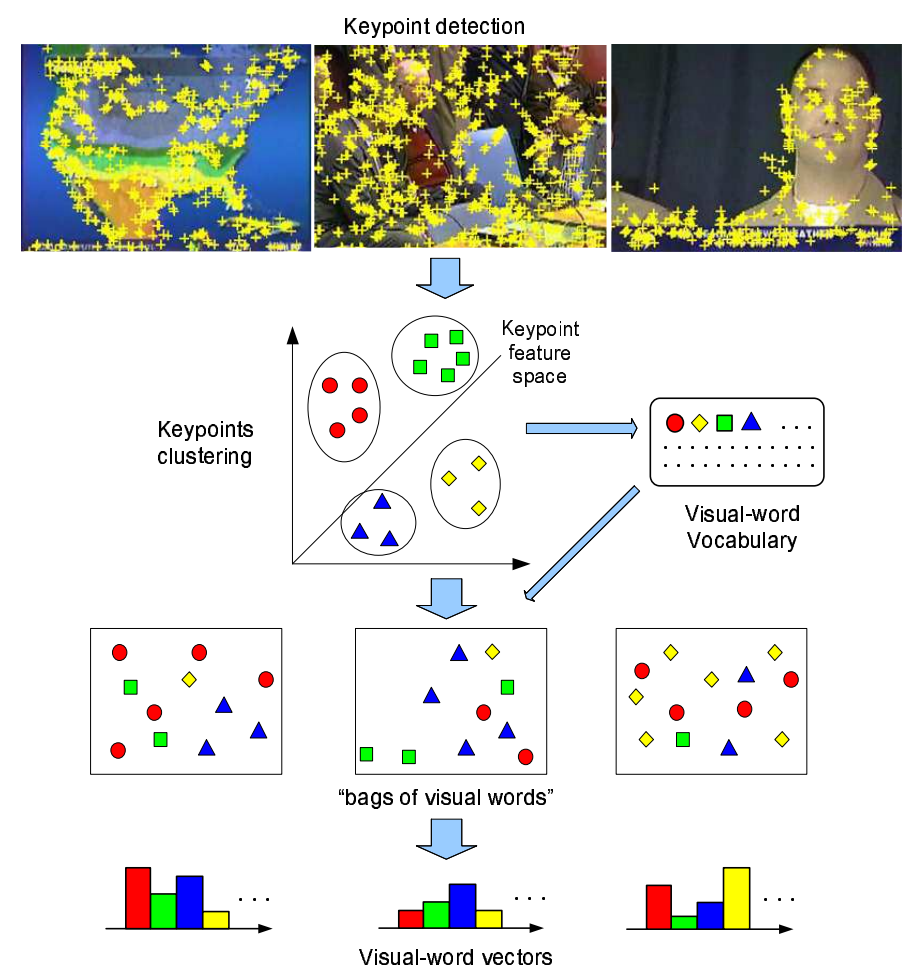

Figure 1: Generating visual-word image representation based on vector-quantized keypoint features

sentation analogous to the bag-of-words representation of text documents. There have been works using this vectorquantized keypoint feature, or bag-of-visual-word representation, for image classification [8, 9, 5, 18, 23, 22]. Our work examines the effectiveness of various representation choices, which is yet to be thoroughly studied in previous work.

Text categorization (TC) is a well studied area in IR. In TC, documents are represented as "bags of words" after stop-word removal and stemming. Each document is described either by a binary vector indicating the presence or absence of terms (e.g., [4]), or by a vector consisting of the $t f$ or $t f$-idf weights of the terms (e.g., [6], [20]). Yang et al. [21] has studied the feature selection methods in TC, and found that up to $98 \%$ of the unique terms in the vocabulary can be eliminated without sacrificing classification accuracy. Different learning algorithms have been applied to TC, including SVM, k-Nearest Neighbor, Naive Bayes, Linear Least Square Fit, which are surveyed in [20] and [4].

\section{BAG-OF-VISUAL-WORDS}

Similar to terms in a text document, an image has local interest points or keypoints defined as salient image patches (small regions) that contain rich local information of the image. Denoted by small crosses in the three images in Figure 1, keypoints are usually around the corners and edges of image objects, such as the edges of the map and around people's faces. We use the Difference of Gaussian (DoG) detector [11] to automatically detect keypoints from images. The detected keypoints are depicted using PCA-SIFT descriptor, which is a 36-dimensional real-valued feature vector [7].

An image can be represented by a set of keypoint descriptors, but this set varies in cardinality and lacks meaningful 
ordering. This creates difficulties for many learning methods (e.g., supervised classifiers) that require feature vectors of fixed dimension as input. In fact, there are novel learning algorithms designed to handle features as sets of unordered vectors with different cardinality, such as the work by Carneiro et al. on multiple instance learning (MIL) [3], and the method proposed by Li and Wang [10]. These algorithms, however, may impose constraints which are not appropriate. For example, a constraint in MIL would be that an image is positive if at least one keypoint is positive, and negative negative if all the keypoints are negative, while in practice it makes no sense to judge whether a keypoint is positive/negative by itself. Moreover, most of the widely used classification algorithms, such as support vector machines (SVMs) [2], still require feature vectors of same length. It is still desirable to transform raw keypoint features into an image feature with a fixed dimension.

To address this problem, we use the vector quantization (VQ) technique which clusters the keypoint descriptors in their feature space into a large number of clusters using the K-means clustering algorithm and encodes each keypoint by the index of the cluster to which it belongs. We conceive each cluster as a visual word that represents a specific local pattern shared by the keypoints in that cluster. Thus, the clustering process generates a visual-word vocabulary describing different local patterns in images. The number of clusters determines the size of the vocabulary, which can vary from hundreds to over tens of thousands. By mapping the keypoints to visual words, we can represent each image as a "bag of visual words". This representation is analogous to the bag-of-words document representation in terms of form and semantics. Both representations are sparse and high-dimensional, and just as words convey meanings of a document, visual words reveal local patterns characteristic of the whole image.

The bag-of-visual-words representation can be converted into a visual-word vector similar to the term vector of a document. The visual-word vector may contain the presence or absence information of each visual word in the image, the count of each visual word (i.e., the number of keypoints in the corresponding cluster), or the count weighted by other factors (see Section 4.3). Visual-word vectors are used in our image classification approach. The process of generating visual-word representation is illustrated in Figure 1.

\section{REPRESENTATION CHOICES}

Once images are represented as bags of visual words, we can classify them in the same way we classify text documents. The general approach is to build supervised classifiers based on visual-word features from labeled images and apply them to predict the labels of other images. There are techniques that can affect the visual-word feature representations and consequently the classification performance. Some of these techniques are borrowed from the area of text categorization, such as term weighting, stop word removal, and feature selection, while others are unique to images, such as changing the vocabulary size and encoding the spatial information. We discuss these techniques below.

\subsection{Vocabulary size}

Unlike the vocabulary of a text corpus whose size is relatively fixed, the size of a visual-word vocabulary is controlled by the number of keypoint clusters in the clustering process.
Choosing the right vocabulary size involves the trade-off between discriminativity and generalizability. With a small vocabulary, the visual-word feature is not very discriminative because dissimilar keypoints can map to the same visual word. As the vocabulary size increases, the feature becomes more discriminative, but meanwhile less generalizable and forgiving to noises, since similar keypoints can map to different visual words. Using a large vocabulary also increases the cost associated with clustering keypoints, computing visualword features, and running supervised classifiers.

There is no consensus as to the appropriate size of a visualword vocabulary. The vocabulary size used in previous work varies from several hundreds $[8,22]$, to thousands and tens of thousands $[18,23]$. Their results are not directly comparable due to the difference on corpus and classification methods. To find out the proper range of vocabulary size, we experiment with vocabularies with sizes varying from 200 to 320,000 . We are also interested in comparing the size of a visual-word vocabulary to that of a text vocabulary, which is usually around thousands to tens of thousands.

\subsection{Stop word removal}

Stop word removal is a standard technique in text categorization. Are there also "visual stop words" that represent local image patterns totally useless for retrieval and classification? Sivic and Zisserman [18] claimed that the most frequent visual words in images are "stop words" and need to be removed from the feature representation. There is however no empirical evidence showing that removing them improves image classification performance. Since it is very difficult to judge whether each visual word is a stop word, we focus on the relationship between the most frequent visual words and the classification performance.

\subsection{Weighting schemes}

Since term weighting is a key technique in IR $[17,1]$, we explore its use in visual-word feature representation. Two major factors in term weighting are $t f$ (term frequency) and $i d f$ (inverse document frequency). A third factor is normalization, which converts the feature into unit-length vector to eliminate the difference between short and long documents. Many text categorization methods use weighting schemes based on these factors, such as "tfc" in [6], "lfc" in [21], while some simply use binary term vectors [4].

We apply popular term weighting schemes in IR to the visual-word feature vectors. These schemes are summarized in Table 1, where they are named after the convention in IR [17]. These schemes are chosen to allow us to study the impact of $t f, i d f$, and the normalization factor on classification performance. Note that $t f_{i}$ is the number of times a visual word $t_{i}$ appears in an image, $N$ is the total number of images in the corpus, and $n_{i}$ is the number of images having visual word $t_{i}$.

We have seen in previous work the use of vectors containing the counts of visual words (which are essentially $t f$ features) for image classification [8,22], and the use of $t f$-idf weighted features for image search $[18,23]$, but no comparisons have been made with other weighting schemes. As we will see, the best weighting scheme in IR does not guarantee good performance in image classification. In particular, the normalization factor, which eliminates the difference on the numbers of keypoints in images, may have a negative effect. Even among images of the same size, the number of 
Table 1: Weighting schemes for visual-word feature

\begin{tabular}{ccc}
\hline Name & Factors & Value for $t_{i}$ \\
\hline bxx & binary & 1 if $t_{i}$ is present, 0 if not \\
txx & $t f$ & $t f_{i}$ \\
txc & $t f$, normalization & $\frac{t f_{i}}{\sum_{i} t f_{i}}$ \\
tfx & $t f$, idf & $t f_{i} \cdot \log \left(N / n_{i}\right)$ \\
tfc & $t f, i d f$, normalization & $\frac{t f_{i} \cdot \log \left(N / n_{i}\right)}{\sum_{i} t f_{i} \cdot \log \left(N / n_{i}\right)}$ \\
\hline
\end{tabular}

keypoints (visual words) varies according to the complexity of the image content. For example, an image showing a complex street scene may have over 1000 keypoints, while an image showing a smooth sky background may have less than 100 keypoints. An image with many keypoints usually has very different content from one with fewer keypoints, even though the relative distribution of their keypoints after being mapped to visual words is similar. Normalization eliminates such difference and makes the two images less distinguishable.

\subsection{Feature selection}

Feature selection is an important technique in text categorization for reducing the vocabulary size and consequently the feature dimension. It uses a specific criterion for measuring the "informativeness" of each word and eliminates the non-informative words. Yang et al. [21] found out that, when a good criterion is used, up to $98 \%$ of the unique words in the vocabulary can be removed without loss of text categorization accuracy. In image classification, feature selection is also important as the size of the visual-word vocabulary is usually very high, but it has not been studied in any previous work. We experiment with five feature selection criteria which are widely used in text categorization [21]:

- document frequency (DF): DF is the number of images (documents) in which a visual word (word) appears. In text categorization, words with small $D F$ are removed since rare words are usually non-informative for category prediction. Not knowing whether frequent visual words or rare ones are more informative for scene classification, we adopt two opposite selection criteria based on $D F$ : DF_max chooses visual words with $D F$ above a predefined threshold, while DF_min chooses visual words with $D F$ below a threshold.

- $x^{2}$ statistics (CHI): The $x^{2}$ statistics measures the level of (in)dependence between two random variables [21]. Here we compute $x^{2}\left(t, c_{i}\right)$ between a specific visual word $t$ and the binary label for an image class $c_{i}$. A large value of $x^{2}\left(t, c_{i}\right)$ indicates a strong correlation between $t$ and $c_{i}$, and vice versa. Since $x^{2}\left(t, c_{i}\right)$ depends on a specific class, we compute the average statistics across a total of $M$ image classes in the corpus as $x_{\text {avg }}^{2}(t)=\frac{1}{M} \sum_{i=1}^{M} x^{2}\left(t, c_{i}\right)$. We then eliminate visual words with $x_{a v g}^{2}(t)$ below a threshold.

- Mutual information (MI): $M I$ is another measure of the dependence between two random variables. The $M I$ between a visual word $t$ and a class label $c$ is:

$$
M I(t, c)=\sum_{t \in\{0,1\}} \sum_{c \in\{0,1\}} P(t, c) \log \frac{P(t, c)}{P(t) P(c)}
$$

We compute $M I_{\text {avg }}(t)=\frac{1}{M} \sum_{i=1}^{M} M I\left(t, c_{i}\right)$, and remove visual words with $M I_{\text {avg }}(t)$ below a threshold.

- Pointwise Mutual information (PMI): PMI is directly related to $M I$. It uses one term in the sum of Eq.(1) to measure the association between a visual word $t$ and a class label $c$ :

$$
P M I(t, c)=\log \frac{P(t=1, c=1)}{P(t=1) P(c=1)}
$$

Visual words with small $P M I_{\text {avg }}(t)$ are eliminated from the vocabulary.

\subsection{Spatial information}

Where within a text document a certain word appears is usually not very relevant to the category this document belongs to. The spatial locations of keypoints in an image, however, carry important information for classifying the image. For example, an image showing a beach scene typically consists of sky-like keypoints on the top and sandslike keypoints at the bottom. The plain bag-of-visual-words representation described in Section 3 ignores such spatial information and may result in inferior classification performance. To integrate the spatial information, we partition an image into equal-sized rectangular regions, compute the visual-word feature from each region, and concatenate the features of these regions into a single feature vector. There can be many ways of partitioning, e.g., $3 \times 3$ means cutting an image into 9 regions in 3 rows and 3 columns.

This region-based representation has its downside in terms of cost and generalizability. Dividing an image into $m \times n$ regions increases the feature dimension by $m \times n$ times, making the feature computationally expensive. Besides, encoding spatial information can also make the representation less generalizable. Suppose an image class is defined by the presence of a certain object, say, airplane, which may appear anywhere in an image. Using region-based representation can cause a feature mismatch if the airplanes in the training images are in different regions from those in the testing images. Another risk is that many objects may cross region boundaries. Based on these considerations, we prefer relatively coarse partitions of image regions to fine-grained partitions.

\section{DATA COLLECTIONS}

We use two corpora to study the bag-of-visual-word representation and its use in image classification: the TRECVID 2005 corpus and the PASCAL 2005 corpus.

The TRECVID corpus contains 34-hour footage of broadcast news video from 6 channels, which was used for TREC Video Retrieval Evaluation 2005 [19]. The video has been segmented into a total of 29,252 shots, and a video frame is extracted from each shot as its keyframe. The data have been annotated with labels of 39 semantic concepts in the LSCOM-Lite project [14]. We rank the 39 concepts by frequency (i.e., the number of shots where the concept is present) and select the 20 most frequent concepts since the rare concepts have insufficient training data. These 20 concepts cover many different types, including outdoor scenes (e.g., waterscape, mountain), indoor scenes (e.g., meeting, studio), objects (e.g., car, computer), people activities (e.g., marching). The goal is to classify the 29,252 video frames according to the presence of any of the 20 semantic concepts. 


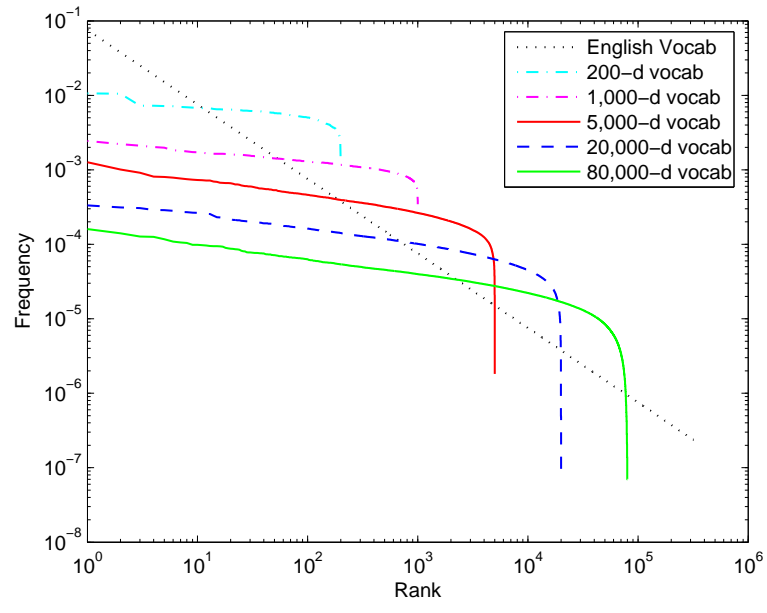

Figure 2: The frequency of visual words from vocabulary of different sizes plot against their frequency ranks in log-log scale.

Note that this is a multi-label corpus in that there can be zero or more than one concept present in a video frame. This is a huge corpus with highly diversified content, as it contains any possible scenes from broadcast news, which makes the classification task very challenging.

The PASCAL corpus was used for the PASCAL Visual Object Classes Challenge 2005. It has 1578 labeled images from multiple sources, which belong to 4 categories as motorbikes, bicycles, people, and cars. Compared with TRECVID, PASCAL is smaller and less diversified, and its images are less noisy and cluttered than the video frames in TRECVID. We choose it since it has been frequently used as a benchmark for evaluating keypoint-based features. Using a second and very different corpus also makes the conclusions in this paper more convincing.

The keypoints in both corpora are detected by the DoG detector [11] and described by the PCA-SIFT descriptor [7]. This results in an average of 490 keypoints per image in TRECVID, and 1,416 keypoints per image in PASCAL. For each corpus, we use the k-means clustering algorithm to cluster a pool of 1,000,000 randomly sampled keypoints into a visual-word vocabulary of any chosen size. The cluster memberships of the remaining keypoints are found using a KD-tree based fast nearest-neighbor search algorithm.

It is interesting to see how the visual words are distributed in an image corpus. Particularly, we want to know whether their distribution satisfies Zipf's law, which is followed by natural languages. Zipf's law says that the frequency of any (visual) word is roughly inversely proportional to its rank in terms of frequency. We choose the TRECVID corpus for this study due to its huge size and diversified content. Under vocabularies of various sizes, we plot the frequency of visual words in TRECVID against their frequency rank in a log-log scale in Figure 2. A Zipf's distribution must be a straight line in such scale. Despite the vocabulary size, we see that every distribution curve starts as a straight line up to a certain point, after which the curve plunges. This shows that, except for those with extremely low frequency, the distribution of visual words basically satisfies Zipf's law. We suspect that the extremely rare words are either noises in images or artifacts of the clustering algorithm, which produces very small clusters.

In Figure 2, the slope of a curve indicates how steep the distribution is. For comparison, we draw an imaginary line to mimic the distribution of a English vocabulary. Obviously, the curves of visual words are not as steep as that of English words, showing that they are distributed more evenly than English words. What is less obvious but equally interesting is that the curve gets steeper as the vocabulary size increases, based on our calculation of the slope. This suggests that the distribution of visual words in a larger vocabulary is more unbalanced.

\section{EXPERIMENT RESULTS}

We study the performance of image classification with different visual-word representations generated using the techniques discussed in Section 4. The TRECVID corpus is partitioned into a training set of 15 -hour footage (15,745 keyframes) and a test set of 18-hour footage (13,507 keyframes). We guarantee that each set has a balanced mixture of data from different channels, and temporally adjacent frames are never assigned to both sets since they are too similar. The PASCAL corpus has been pre-divided into a training, validation, and test set, and we use the first two sets for training and the third for testing.

The classification is conducted in an "one-against-all" manner. Based on Support Vector Machines (SVM) [2], we build 20 binary classifiers for the 20 semantic concepts in TRECVID, and 4 binary classifiers for the 4 object categories in PASCAL, where each classifier is for determining the presence of a specific concept or object. We use average precision (AP) to evaluate the result of a single classifier, and mean average precision (MAP) to aggregate the performance of multiple classifiers. In the following, we examine the impact of various representation choices of visualword features on the classification performance, and compare their performance to that of conventional color/texture features.

\subsection{Vocabulary size}

Figure 3 shows the relationship between the classification performance and the size of a visual-word vocabulary. We use binary features ("bxx" in Table 1) without spatial information or feature selection. Both the linear and RBF kernel are used in SVM. For the RBF kernel, different choices of the gamma parameter are tried and the best result is reported.

We see that on both corpora, as the vocabulary size increases from 200 to over 80,000 , the performance first rises dramatically, peaks at certain points, and after that either levels off or drops mildly. Although it is not surprising to see such pattern, the important thing revealed by this experiment is the range of optimal vocabulary sizes, which are larger than the vocabulary sizes seen in most previous work. The optimal vocabulary size is around 20,000 to 80,000 for TRECVID, and around 5,000 for PASCAL, both comparable to the size of a typical text vocabulary which is around thousands or tens of thousands. The difference between the two corpora can be explained by the fact that the keypoints in the smaller PASCAL corpus are not as widely-spread as those in TRECVID, and therefore demands fewer clusters (visual words). Although the optimal vocabulary size is clearly corpus dependent, this experiment suggests the use of relatively large vocabularies. 

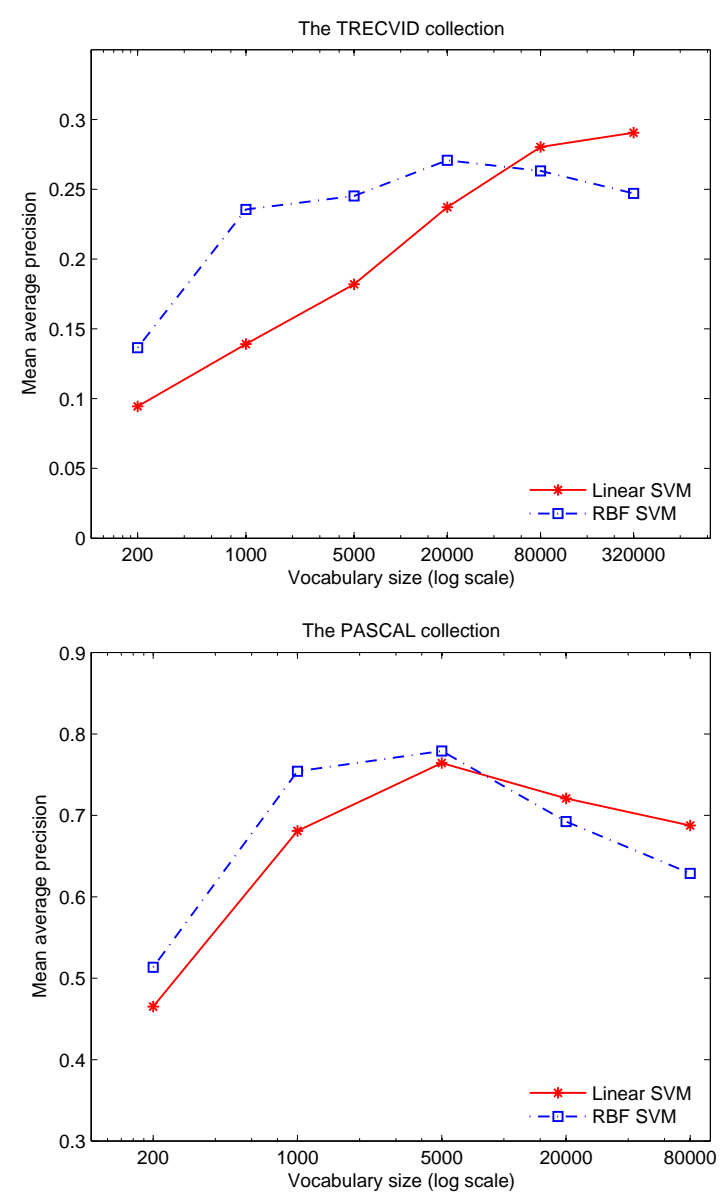

Figure 3: The classification performance at different vocabulary sizes on TRECVID and PASCAL. (Note that the $\mathrm{x}$-axis is in log scale.)

Another interesting observation comes from the comparison between the two kernels of SVM. For small vocabularies, the RBF kernel has a clear advantage over the linear one, but this advantage is reversed after the peak performance is reached. This suggests that the visual words in a small vocabulary are highly correlated, but become more independent and gain the property of linear separability as the vocabulary gets larger. When the visual words are independent, kernels that consider inter-feature correlations (e.g., RBF) have no advantage over linear kernels and may perform poorly due to overfitting.

\subsection{Stop word removal}

Do the most frequent visual words function like "stop words"? We approach this problem by examining the classification performance using vocabularies without the most frequent visual words, where the word frequency is computed from each corpus. As shown in Table 2, removing the most frequent words causes a small but steady decrease of performance on both corpora. This shows that these frequent visual words are unlikely stop words, since removing stop words should improve the classification performance. While it is premature to say there are no visual stop words, we show that eliminating the most frequent visual words is not desirable, which is against the claim in [18].

\begin{tabular}{|c|c|ccccc|}
\hline \multirow{2}{*}{ Corpus } & Whole & \multicolumn{5}{|c|}{ Percent of removed words } \\
\cline { 3 - 7 } & Vocab & $0.5 \%$ & $1 \%$ & $3 \%$ & $5 \%$ & $10 \%$ \\
\hline TRECVID & 0.280 & 0.279 & 0.278 & 0.275 & 0.273 & 0.267 \\
PASCAL & 0.778 & 0.778 & 0.777 & 0.775 & 0.773 & 0.771 \\
\hline
\end{tabular}

Table 2: The classification performance after removing the most frequent visual words

\subsection{Weighting schemes}

Now we move on to the problem of weighting schemes. Table 3 summaries the classification performance using visualword features weighted by the 5 weighting schemes in Table 1. We use no spatial partitioning or feature selection in this experiment, but vary the vocabulary size to study their relationship with weighting schemes.

First, we focus on the comparison between binary ("bxx") and $t f$ feature ("txx") to see whether the counts of visual words are more informative than their presence or absence. It is only when the vocabulary size drops to 200 that $t f$ features consistently outperform binary features. For larger vocabularies, $t f$ features are (slightly) worse than binary ones in most settings. This observation can be explained from two aspects. For one thing, as the vocabulary gets larger, the count of most visual words is either 0 or 1 and therefore $t f$ features are not much different from binary ones. On the other hand, the count information can be noisy. Suppose a certain visual word is typical among "building" images. An image containing 100 of this visual word is not necessarily more likely to be a "building" than an image containing only 20 of this visual word, but a classifier trained from the first image can be misled by the high count and classify the second image as "non-building". This explains why the count of visual words may not be as effective as their presence/absence information.

Next, we examine the impact of the $i d f$ factor by comparing the performance of "txx" and "tfx". There is no consistent benefit of using $i d f$, as "tfx" (which includes $i d f$ ) is better than "txx" in about half of the settings but worse in the other half. We attribute this to the fact that a discriminative classifier like SVMs can implicitly weight features to achieve maximum data separation, and its weighting strategy is presumably a better one than the heuristic $i d f$ method. So weighting scheme is discouraged when a powerful classifier such as SVMs is used.

We have contradicting observations regarding the normalization factor between the two corpora. In PASCAL, "txc" (normalized) consistently outperforms "txx" (unnormalized), and "tfc" (normalized) outperforms "tfx" (unnormalized) in all but one setting. However, in TRECVID the un-normalized features are always better than their normalized counterparts. A plausible explanation is that, PASCAL has images of different sizes, and its classification performance benefits from the normalization factor which eliminates the difference on image sizes. This is not the case with TRECVID, which contains video frames of identical size. Normalization hurts the performance by suppressing the difference on the number of keypoints in each video frame.

Overall, using binary visual-word features is a good choice which always produce top or close-to-top performance in most of our experiment settings. This is especially true when a large vocabulary is used, which is likely to be the case if classification accuracy is the major consideration. 


\begin{tabular}{|l|l|ccccc|ccccc|}
\hline \multirow{2}{*}{ Corpus } & Vocabulary & \multicolumn{6}{|c|}{ Linear SVM } & \multicolumn{5}{c|}{ RBF SVM } \\
\cline { 3 - 11 } & size & $b x x$ & $t x x$ & $t x c$ & $t f x$ & $t f c$ & $b x x$ & $t x x$ & $t x c$ & $t f x$ & $t f c$ \\
\hline \hline \multirow{3}{*}{ TRECVID } & 200 & 0.095 & $\mathbf{0 . 1 5 2}$ & 0.109 & 0.147 & 0.110 & 0.137 & $\mathbf{0 . 1 6 7}$ & 0.112 & 0.130 & 0.108 \\
& 1,000 & 0.139 & 0.162 & 0.137 & $\mathbf{0 . 1 8 3}$ & 0.142 & $\mathbf{0 . 2 3 5}$ & 0.202 & 0.141 & 0.161 & 0.128 \\
& 5,000 & 0.183 & 0.178 & 0.150 & $\mathbf{0 . 2 0 5}$ & 0.153 & $\mathbf{0 . 2 4 5}$ & 0.224 & 0.141 & 0.194 & 0.145 \\
& 20,000 & $\mathbf{0 . 2 3 7}$ & $\mathbf{0 . 2 2 8}$ & 0.185 & 0.225 & 0.188 & $\mathbf{0 . 2 7 1}$ & $\mathbf{0 . 2 7 8}$ & 0.163 & 0.216 & 0.184 \\
\hline \hline \multirow{3}{*}{ PASCAL } & 200 & 0.465 & 0.680 & 0.605 & 0.639 & $\mathbf{0 . 6 9 3}$ & 0.513 & 0.670 & $\mathbf{0 . 7 4 2}$ & 0.619 & 0.686 \\
& 1,000 & $\mathbf{0 . 6 8 1}$ & 0.677 & 0.677 & $\mathbf{0 . 6 9 0}$ & $\mathbf{0 . 6 8 3}$ & $\mathbf{0 . 7 5 4}$ & 0.639 & 0.751 & 0.618 & 0.722 \\
& 5,000 & $\mathbf{0 . 7 6 4}$ & 0.738 & 0.745 & 0.740 & 0.745 & $\mathbf{0 . 7 7 7}$ & 0.708 & 0.737 & 0.757 & 0.734 \\
& 20,000 & $\mathbf{0 . 7 2 1}$ & 0.682 & 0.708 & 0.682 & 0.711 & $\mathbf{0 . 6 8 3}$ & 0.642 & $\mathbf{0 . 6 9 0}$ & 0.528 & $\mathbf{0 . 6 8 2}$ \\
\hline
\end{tabular}

* Weighting: $b x x=$ binary, $t x x=t f, t x c=t f+$ normalization, $t f x=t f+i d f, t f c=t f+i d f+$ normalization

Table 3: The classification performance (MAP) on TRECVID and PASCAL corpus under different weighting schemes and vocabulary sizes. The bold font indicates the top performers in each setting.
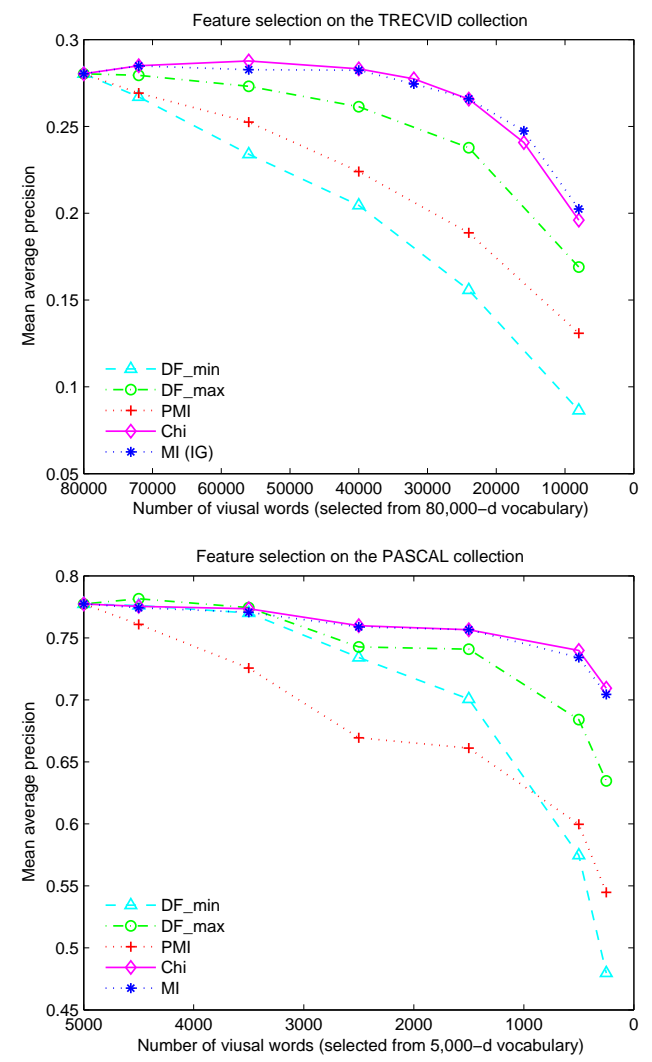

Figure 4: Classification performance under vocabularies pruned using various feature selection criteria.

\subsection{Feature selection}

We examine feature selection techniques on the best vocabulary for each corpus, i.e., a 80,000-d vocabulary for TRECVID and a 5,000-d vocabulary for PASCAL. The 5 feature selection criteria discussed in Section 4.4 are compared, which are DF-max, DF-min, CHI, MI, and PMI. We reduce the vocabulary size to several percentages of its original size $(90 \%, 70 \%, \ldots, 10 \%)$ by removing the most uninformative words determined by each criterion, and evaluate the classification performance in each setting. The results are shown in Figure 4.

We see that when effective criteria like $M I$ and $C H I$ are used, there is only minimum loss of MAP when the vocabu- lary is reduced by half. When the vocabulary is reduced by $70 \%$, the MAP has dropped merely by $5 \%$, but after that it drops much faster. This shows that feature selection is an effective technique in image classification. In comparison, in text categorization a vocabulary can be reduced by up to $98 \%$ without loss of classification accuracy [21], which implies that the percentage of uninformative terms in text documents is much larger than in images.

Among different feature selection methods, $C H I$ and $M I$ are top performers on both corpora, followed by DF_max, while the performance of DF_min and $P M I$ are lower than the others. This order is basically consistent with that in the text categorization $[21]^{1}$. The fact that DF_max is significantly better than DF_min implies that frequent visual words widely spread among images are more informative than rare visual words in terms of discriminative power. This is consistent with the finding in text categorization that frequent words (not including stop words) are more informative than rare words [21]. It also partially explains why the feature selection can be done more aggressively on text documents than on images. As shown in Figure 2, the distribution of text words is much more uneven than that of visual words, which means there is a larger percentage of un-informative rare words to be eliminated from a text vocabulary.

\subsection{Spatial information}

The importance of spatial information can be seen by comparing the classification performance between plain visualword features computed from whole images and features computed from image regions. We examine 4 ways of partitioning image regions, including $1 \times 1$ (the whole image), $2 \times 2$ ( 4 regions), $3 \times 3$ ( 9 regions), and $4 \times 4$ (16 regions). Figure 5 shows the classification performance on both corpora using different spatial partitions and vocabulary sizes. For each setting, we experiment with both the linear and RBF kernel of SVM, and the performance of the better one is reported.

We see that the spatial information substantially improves the classification performance when the vocabulary is small. With a 200 -d vocabulary, as the partition changes from $1 \times 1$ to $4 \times 4$, MAP doubles on TRECVID and increases by $60 \%$ on PASCAL. This agrees with the results in [8] that spatial information achieved significant improvement on texture and object categorization with small vocabularies of size 16 ,

\footnotetext{
${ }^{1}$ By definition, $M I$ in this paper is equivalent to $I G$ in [21], while $P M I$ here is equal to $M I$ in that paper.
} 

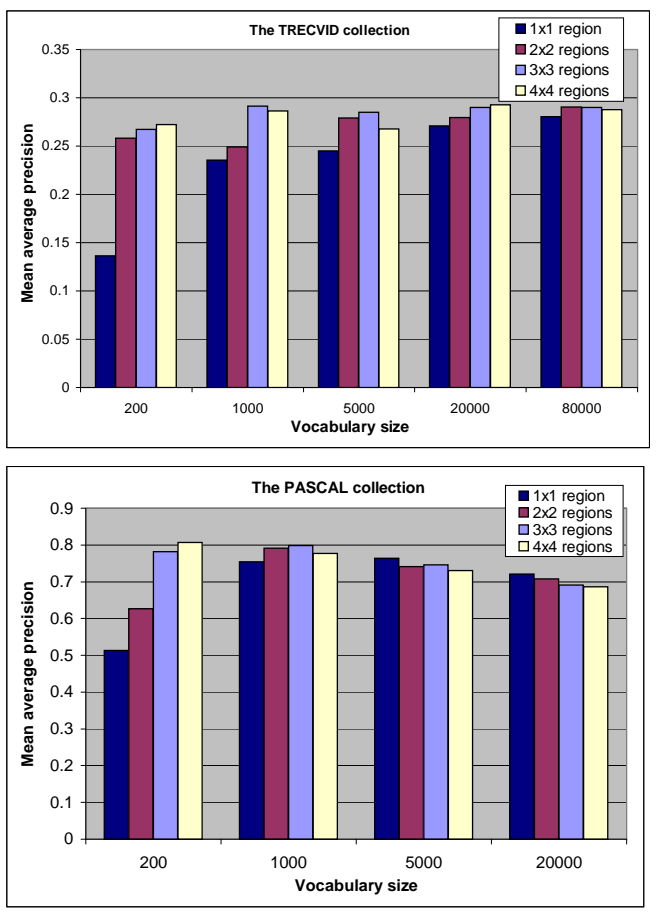

Figure 5: Classification performance of region-based features computed from different spatial partitions

200 and 400. An observation not covered in [8] but obvious in our experiment, is that the improvement from the spatial information diminishes as the vocabulary sizes increases. In Figure 5, the spatial partitioning is of little help after the vocabulary sizes reaches 20,000 in TRECVID, and it even hurts the performance of PASCAL after the vocabulary size reaches 5,000. This shows the contributions from a large vocabulary and from spatial information are not orthogonal and not even very complementary, which is slightly counterintuitive. We find in Figure 5 that the peak performance can be reached either by increasing the vocabulary size or by using more fine-grained spatial partition, but combining large vocabulary with spatial partitioning fails to push the performance further. This agrees with the claim of Nister and Stewenius [15] that an extremely large vocabulary achieves good performance without using geometric information. A plausible explanation is that, when the vocabulary size is small, dissimilar keypoints in different regions can be mapped into the same visual word, but using spatial information helps discriminate them and therefore improves the classification performance. When the vocabulary size is large enough, keypoints are well discriminated and adding spatial information hardly further discriminate them

As to the choice of appropriate partitions, both $3 \times 3$ and $4 \times 4$ appear to be reasonable choices because either of them is the best performer at various vocabulary sizes. There seems to be no need to go beyond $4 \times 4$, since after that the performance levels off or slightly drops. This is also consistent with the results in [8] where $4 \times 4$ is better than either coarser or more fine-grained partitions on different data sets. Overall, using a small vocabulary (e.g., 200-d or 1,000 -d) with $3 \times 3$ or $4 \times 4$ partition is a good configuration which achieves top or close-to-top performance and is less expensive than using a larger vocabulary.

\subsection{Combining with color/texture features}

Besides the use of local, keypoint-based features, image and video classification is more often done based on global image features such as color histograms and color moments, texture features based on wavelet or Gabor filters, etc. While keypoint features describe the local structures in an image and do not contain color information, global features are statistics about the overall distribution of color, texture, or edge information in the image. Hence, we expect these two types of features are complementary for scene classification, which requires either global color information (e.g., for "Sky", "Snow"), or local structure information (e.g., for "Building", "Car"), or both (e.g., for "Studio"). It is interesting not only to compare the performance of the two features, but also to see whether their combination further improves the performance.

We experiment with three types of global features: 225d color moment feature computed from a $5 \times 5$ image grids, 48-d Gabor texture feature, and a 273-d feature concatenated from them. We compare their performance with that of local features of various dimensions computed from the whole images or from $3 \times 3$ grids in TRECVID. (We did not perform this comparison on PASCAL, where keypoint features are clearly more effective since they are used by most top-performing methods.) The combination of a local feature and a global feature is done in a "late fusion" fashion, where separate classifiers are built on two features and the final score for an image is a weighted sum of the outputs of two classifiers. The combination weights are learned on a held-out set using logistic regression.

Two observations can be made from the results shown in Table 4 and 5. First, carefully engineered local features produce comparable performance to good global features. For example, $1 \times 1$ local feature on 80,000 -d vocabulary, or $3 \times 3$ local feature on 1,000 -d vocabulary outperforms color moment or Gabor texture feature, and is comparable to their combination. Second, combining a local feature with a global one furthers the performance by $10-20 \%$ over the higher one of the two. The highest performance (MAP $=$ 0.349 ) is achieved by combining the color moment feature, Gabor texture feature, and $3 \times 3$ local feature on a 20,000 vocabulary. This shows that these two types of features carry complementary information for classification, and should be used together for good performance.

\section{CONCLUSION}

Bag-of-visual-word is an effective image representation in the classification task, but various representation choices w.r.t its dimension, weighting, and selection of visual words has not been thoroughly examined. In this paper, we have applied techniques used in text categorization, including term weighting, stop word removal, feature selection, to generate various visual-word representations, and studied their impact to classification performance on the TRECVID and PASCAL collections. This study provides an empirical basis for designing visual-word representation that is likely to produce good classification performance.

The analogy between visual words in images and words in documents opens up opportunities for migrating techniques of information retrieval (IR) to solve problems in image and video data. Given the success on the classification task, we plan to apply IR techniques to image and video search based 


\begin{tabular}{|c|c|c|c|c|c|c|c|}
\hline & \multicolumn{5}{|c|}{ Visual words feature $(1 \times 1$ partition $)$} \\
\hline & & & $200-\mathrm{d}$ & $1,000-\mathrm{d}$ & $5,000-\mathrm{d}$ & $20,000-\mathrm{d}$ & $80,000-d$ \\
\hline & & & 0.137 & 0.235 & 0.245 & 0.271 & 0.280 \\
\hline Global & Color & 0.250 & 0.252 & 0.305 & 0.310 & 0.316 & 0.328 \\
\hline Fea- & Gabor & 0.182 & 0.212 & 0.265 & 0.278 & 0.286 & 0.303 \\
\hline ture & Color+Gabor & 0.292 & 0.300 & 0.323 & 0.327 & 0.329 & 0.343 \\
\hline
\end{tabular}

Table 4: The MAP of global features, local features based on $1 \times 1$ grids, and their combinations in TRECVID.

\begin{tabular}{|c|c|c|c|c|c|c|c|}
\hline & \multicolumn{5}{|c|}{ Visual words feature $(3 \times 3$ partition $)$} \\
\hline & & & $200-d$ & $1,000-\mathrm{d}$ & $5,000-d$ & $20,000-d$ & $80,000-d$ \\
\hline & & & 0.267 & 0.291 & 0.285 & 0.289 & 0.290 \\
\hline$\overline{\text { Global }}$ & Color & 0.250 & 0.295 & 0.301 & 0.321 & 0.334 & $\overline{0.334}$ \\
\hline Fea- & Gabor & 0.182 & 0.273 & 0.293 & 0.286 & 0.291 & 0.315 \\
\hline ture & Color+Gabor & 0.292 & 0.318 & 0.329 & 0.339 & 0.349 & 0.349 \\
\hline
\end{tabular}

Table 5: The MAP of global features, local features based on $3 \times 3$ grids, and their combinations in TRECVID.

on the bag-of-visual-words representation. While there has been some pilot works on this direction [18, 23], a thorough study of this approach is missing. More interesting future work is to build "visual language models" that describe the distribution of visual words in images. Such visual language models provide a generative view of images, and can be used for image retrieval and classification using existing language modeling techniques for IR [16]. We can even build bigram or trigram type of models of visual words to capture the spatial relationships of adjacent keypoints, which could be more powerful in terms of describing complex image content.

\section{REFERENCES}

[1] R. Baeza-Yates and B. Ribeiro-Neto. Modern Information Retrieval. ACM Press Series/Addison Wesley, 1999.

[2] C. J. C. Burges. A tutorial on support vector machines for pattern recognition. Data Mining and Knowledge Discovery, 2(2):121-167, 1998.

[3] G. Carneiro, A. B. Chan, P. J. Moreno, and N. Vasconcelos. Supervised learning of semantic classes for image annotation and retrieval. IEEE Trans. Pattern Anal. Mach. Intell., 29(3):394-410, 2007.

[4] S. Dumais, J. Platt, D. Heckerman, and M. Sahami. Inductive learning algorithms and representations for text categorization. pages 148-155, 1998.

[5] Y.-G. Jiang, C.-W. Ngo, and J. Yang. Towards optimal bag-of-features for object categorization and semantic video retrieval. In Proc. of ACM Int'l Conf. on Image and Video Retrieval, 2007.

[6] T. Joachims. Text categorization with suport vector machines: Learning with many relevant features. In Proc. of the 10th European Conf. on Machine Learning, pages 137-142. Springer-Verlag, 1998.

[7] Y. Ke and R. Sukthankar. Pca-sift: A more distinctive representation for local image descriptors. In Proc. of the IEEE Conf. on Computer Vision and Pattern Recognition, 2004.

[8] S. Lazebnik, C. Schmid, and J. Ponce. Beyond bags of features: Spatial pyramid matching for recognizing natural scene categories. In Proc. of 2006 IEEE
Computer Society Conf. on Computer Vision and Pattern Recognition, volume 2, pages 2169-2178, 2006.

[9] F.-F. Li and P. Perona. A bayesian hierarchical model for learning natural scene categories. In Proc. of the 2005 IEEE Computer Society Conf. on Computer Vision and Pattern Recognition, pages 524-531, 2005.

[10] J. Li and J. Z. Wang. Real-time computerized annotation of pictures. In Proc. of the 14th Annual ACM Int'l Conf. on Multimedia, pages 911-920, 2006.

[11] D. G. Lowe. Distinctive image features from scale-invariant keypoints. Int. J. Comput. Vision, 60(2):91-110, 2004.

[12] K. Mikolajczyk and C. Schmid. Scale and affine invariant interest point detectors. Int. J. Comput. Vision, 60(1):63-86, 2004.

[13] K. Mikolajczyk and C. Schmid. A performance evaluation of local descriptors. IEEE Transactions on Pattern Analysis and Machine Intelligence, 27(10):1615-1630, 2005.

[14] M. R. Naphade, L. Kennedy, J. R. Kender, S. F. Chang, J. Smith, P. Over, and A. Hauptmann. A light scale concept ontology for multimedia understanding for TRECVID 2005. In IBM Research Technical Report, 2005.

[15] D. Nister and H. Stewenius. Scalable recognition with a vocabulary tree. In Proc. of 2006 IEEE Computer Society Conf. on Computer Vision and Pattern Recognition, pages 2161-2168, Los Alamitos, CA, USA, 2006.

[16] J. M. Ponte and W. B. Croft. A language modeling approach to information retrieval. In Research and Development in Information Retrieval, pages 275-281, 1998.

[17] G. Salton and C. Buckley. Term-weighting approaches in automatic text retrieval. Information Processing and Management: an Int'l Journal, 25(5):513-523, 1988.

[18] J. Sivic and A. Zisserman. Video google: A text retrieval approach to object matching in videos. In Proc. of 9th IEEE Int'l Conf. on Computer Vision, Vol. 2, 2003. 
[19] A. Smeaton and P. Over. Trecvid: Benchmarking the effectiveness of infomration retrieval tasks on digital video. In Proc. of the Intl. Conf. on Image and Video Retrieval, 2003.

[20] Y. Yang and X. Liu. A re-examination of text categorization methods. In Proc. of the 22nd Annual int'l ACM SIGIR Conf. on Research and Development in Information Retrieval, pages 42-49, 1999.

[21] Y. Yang and J. Pedersen. A comparative study on feature selection in text categorization. In 14th Int'l Conf. on Machine Learning, pages 412-420, 1997.
[22] J. Zhang, M. Marszalek, S. Lazebnik, and C. Schmid. Local features and kernels for classification of texture and object categories: An in-depth study. In Technical report, INRIA, 2005.

[23] W. Zhao, Y.-G. Jiang, and C.-W. Ngo. Keyframe retrieval by keypoints: Can point-to-point matching help? In Proc. of 5th Int'l Conf. on Image and Video Retrieval (CIVR), pages 72-81, 2006. 